\title{
A proof theory of asynchronously communicating sequential processes
}

F. S. de Boer ${ }^{1}$, N. France ${ }^{2}$, M. van Hulst ${ }^{3}$, F. A. Stomp ${ }^{4}$

${ }^{1}$ Utrecht University, ${ }^{2}$ The Technion, ${ }^{3}$ Intel Corporation, ${ }^{4}$ University of California

${ }^{1}$ Department of Computer Science, P.O. Box 80089, 3508 TB Utrecht, The Netherlands. frankb@cs.ruu.nl.

${ }^{2}$ Department of Computer Science, Haifa 32000, Israel. francez@cs.technion.ac.il.

${ }^{3}$ Santa Clara, CA 95052-8119, USA. mvanhuls@mipos2.intel.com.

${ }^{4}$ Department of Computer Science, Davis, CA 95616, USA. stomp@cs.ucdavis.edu.

\begin{abstract}
We present a compositional Hoare logic for distributed systems in which communication is asynchronous via unbounded FIFO channels. Our main result is that the noncompositional proof method for (synchronous) CSP of Apt, Francez, and de Roever applied to asynchronous communication allows a simple compositional formulation in which the so-called cooperation test can be fully incorporated in the local verification of the (sequential) components of the system. Thus, the resulting method enables (verified) local specifications of the components, which may be augmented with auxiliary variables, to be combined into a global specification without any test involving the way those local specifications have been derived.

The applicability of our approach is demonstrated by a correctness proof of a distributed program for leader election.
\end{abstract}

\section{Keywords}

Asynchronous communication, compositional reasoning, distributed computing

\section{INTRODUCTION}

Hoare logics have been developed for and applied successfully to a variety of sequential programming constructs (see for an overview [1]). For the parallel programming language CSP a Hoare logic is presented in [3]. The proof 
method in [3] is based on the proof-theoretical ideas first introduced in [18] for a parallel programming language based on communication via shared variables. Characteristic of this method is its two-leveled structure: In the first level the (sequential) components of a system are verified using assumptions about the communication statements; these assumptions in general involve auxiliary variables. The second level consists of checking whether the assumptions introduced in the first level for the various components are mutually consistent with respect to a global invariant. This test is called the cooperation test. Since this test involves information of the internal structure of the local proofs of the correctness of the sequential components, this proof method is not compositional.

\section{The results}

In this paper we develop a compositional Hoare logic for distributed systems composed of processes which communicate asynchronously via unbounded FIFO (First In First Out) channels.

Our main result is that the noncompositional proof method of [3] applied to ACSP (Asynchronously Communicating Sequential Processes) allows a formulation in which the cooperation test can be fully incorporated in the verification of the (sequential) components of a system. More specifically, compositionality is obtained by the introduction of a generalization of the usual Hoare logic for sequential programs. This generalization consists of the addition of a global invariant to local specifications. Such generalized Hoare triples involve a rely/guarantee style of reasoning ([13]) and as such are to be distinguished from the specification formalism of I-logic ([20]). The verified local specifications then can be logically combined into a global specification, without any additional test involving the way these local specifications are derived (as usually codified in proof outlines). As such the resulting proof method is compositional (assuming the following definition: A proof system is called compositional if the specification of a compound programming construct can be derived from specifications of its constituent parts without any further information about the structure of these parts). The applicability of the proof method is demonstrated by proving correctness of a distributed leader election algorithm.

We prove in the full paper that the method is sound and (relative) complete. To obtain a (relative) complete proof method for ACSP auxiliary variables are needed to describe and reason about dynamic control properties.

\section{Comparison with related work}

The first proof method for ACSP was proposed in [22] and [23]. That method extends the system presented in [17] for synchronous CSP. These systems 
are not compositional because of the tests for satisfaction and interferencefreedom. A proof method in the same spirit has been proposed by Camp, Kearns, and Ahuja [9] to reason about communication through flush-channels, which generalize conventional asynchronous channels.

Compositional Hoare logics for CSP have been developed in [24] and [26]. The Hoare logic of [26] has been applied to ACSP in [20]. Other similar compositional proof systems for reasoning about asynchronous communication have been presented in $[6,14,25,21]$. Francez [10] gives on overview of many existing proof methods for sequential and parallel/distributed programs. Compositionality in the above-mentioned systems is achieved by the incorporation of certain logical variables which range over histories, i.e. sequences of communication events. Characteristic of our method on the other hand is that it allows compositional reasoning based on arbitrary auxiliary variables. In other words, it does not force the user (of the method) to use only one particular kind of auxiliary variables like history variables. Instead, our method allows to verify a concurrent system in terms of auxiliary variables which are appropriate for the specification of the particular correctness problem considered. Since in general histories complicate the reasoning process while in many real-life examples simple boolean 'flags' as auxiliary variables already suffice, compositional proof methods, which allow auxiliary variables of any kind, like the one presented in this paper, are clearly more flexible and thus more useful in practice.

The generalization of Hoare logic introduced in this paper is based on the specification of the reactive behaviour of a component of a concurrent system in terms of a global invariant. In [15] and [16] a generalization of Hoare logic has been developed which is also based on the use of invariants in the specification and verification of concurrent systems. The logic of [15] however involves the use of built-in location variables and consequently it does not provide for a more flexible use of arbitrary auxiliary variables. Moreover, in this paper we present a formal semantic justification of generalized Hoare logic in terms of reactive sequences. Such sequences have been introduced in [4] as a general compositional model for concurrent systems based on asynchronous communication. The formal semantic justification of our generalization of Hoare logic allows for simple proofs of soundness and completeness, which are given in the full paper. Basically these proofs generalize in a straightforward manner the corresponding proofs of soundness and completeness (see [1]) of the standard Hoare logic of sequential programs.

\section{The plan of the paper}

In the next section we introduce our programming language of asynchronously communicating sequential processes. We present our proof system for reasoning about partial correctness properties of programs in Section 3. In Section 4, 
we prove correctness of a distributed leader election program. Finally, Section 5 draws some conclusions.

\section{PROGRAMMING LANGUAGE}

In this section we define the syntax and the semantics of the programming language. The semantics describes the behaviour of asynchronously communicating processes. Processes interact only via directed communication channels which are implemented by unbounded FIFO-buffers. A process can send a value along a channel or it can receive a value along a channel. The value sent along a channel is appended to the buffer which implements that channel, whereas receiving a value along a channel consists of retrieving the first element from its corresponding buffer. Thus values are received along a channel in the same order as they have been sent along that channel. A process is suspended when it tries to receive a value along an empty channel. Since buffers are assumed to be unbounded sending values can always take place.

\subsection{Syntax}

We assume given a set Pvar of (program) variables, and a set $C$ of channel names with typical element $c, \ldots$. The sets $P v a r$ and $C$ are assumed to be disjoint.

The syntax of a statement, which can be executed by a (sequential) process, is defined by the following grammar:

$$
S::=x:=e|c ! ! e| c ? ? x\left|S_{1} ; S_{2}\right| \rrbracket_{i}\left[g_{i} \rightarrow S_{i}\right] \mid \star \rrbracket_{i}\left[g_{i} \rightarrow S_{i}\right]
$$

In the above, $e$ denotes an expression, and $A$ denotes some fixed and finite set (of indices.) (The syntactic structures of expressions and of indices are left unspecified.)

The assignment statement $x:=e$ has its usual meaning (e.g. we assume that the execution of an assignment terminates properly). Sending the value of expression $e$ along channel $c$ is described by the send-statement $c ! ! e$, whereas receiving a value along channel $c$ and recording that value in variable $x$ is described by the receive-statement $c ? ? x$. We sometimes refer to a receive- or a send-statement as an IO statement. Sequential composition is denoted by ';'. The statement $\rrbracket_{i}\left[g_{i} \rightarrow S_{i}\right]$ is called a guarded conditional. It consists of a (finite number of) guarded statements $g_{i} \rightarrow S_{i}$. Every guard is either a boolean expression $b$ or a sequential composition $b ; c ? ? x$, for some boolean condition $b$ and some receive-statement $c ? ? x$. (The syntactic structure of boolean expressions is left unspecified.) Guard $b$ is enabled in some state, if it evaluates to true in that state. If enabled, execution of such a guard is equivalent to the execution of a skip-statement. Guard $b ; c ? ? x$ is enabled in some state, if 
in that state $b$ evaluates to true and channel $c$ is not empty. If enabled, execution of such a guard is equivalent to the execution of $c ? ? x$. The execution of a guarded conditional $\rrbracket_{i}\left[g_{i} \rightarrow S_{i}\right]$ consists of the execution of some enabled guard $g_{i}$ and the execution of $S_{i}$ thereafter. If there exists no enabled guard, then the execution of the guarded conditional suspends. The guarded iteration $\star \rrbracket_{i}\left[g_{i} \rightarrow S_{i}\right]$ consists of repeatedly executing the guarded selection $\rrbracket_{i}\left[g_{i} \rightarrow S_{i}\right]$ until all the boolean parts of the guards are false (and the iteration terminates). Note that we do not have send-statements in guards. However this does not reduce the expressive power as in CSP ([8]) because the execution of a send-statement does not depend on the environment.

In order to reason about programs, we may possibly have to expand them by (assignments to) auxiliary variables. These variables are not allowed to affect the flow of control of the original program. They are used only to reason about dynamic control properties. Related with the notion of auxiliary variables is that of a bracketed section. Bracketed sections are used to render the sequential composition of an IO-statement and an assignment to an auxiliary variable atomic, so that it is irrelevant whether an invariant holds in between. The formal definition of this notion can be found in the full paper and is similar to the corresponding notion as defined in [3].

Definition 1 A parallel program is of the form $\left[S_{1}\|\ldots\| S_{n}\right]$ with the following restrictions: The statements $S_{i}$ do not share program variables, channels are unidirectional and connect exactly one sender and one receiver. Formally we assume a partitioning of the set Pvar of program variables into sets Pvar $_{i}$ (here $i$ ranges over the natural numbers.) Also, we assume a partitioning of the set IOvar $=\{c$ ??, c!! | $c \in C\}$ of input/output variables into sets IOvar . $_{\text {. }}$ Let $P \operatorname{var}(S)$ be the set of program variables of $S$ and let $\operatorname{IOvar}(S)$ be the set of those input/output variables for which there occurs a corresponding input/output statement in $S$, e.g. IOvar $(c ? ? x)=\{c ? ?\}$. We then impose on any program $P=\left[S_{1}\|\ldots\| S_{n}\right]$ the syntactic restriction that $\operatorname{Pvar}\left(S_{i}\right) \subseteq P \operatorname{Var}_{i}$ and $\operatorname{IOvar}\left(S_{i}\right) \subseteq \operatorname{IOvar}_{i}$.

\subsection{Semantics}

We define the semantics of the programming language in terms of a structured operational semantics. We first define the notion of a state.

Definition 2 A state assigns values to all program variables and all input/output variables. Define $\Sigma=($ Pvar $\cup I O v a r) \rightarrow\left(V_{a l} \cup V_{a l}{ }^{*}\right)$, where Val denotes some domain of values and $\mathrm{Val}^{*}$ denotes the set of all finite sequences over Val. We impose the restriction that program variables are mapped to values; and that input/output variables are mapped to sequences of values. Elements of $\Sigma$ are called states and denoted by $\sigma, \ldots$. 
The idea is that $\sigma(c ? ?)$ denotes the sequence of values received along channel $c$, whereas $\sigma(c ! !)$ denotes the sequence of values sent along channel $c$.

Definition 3 The transition relation $S \stackrel{\left\langle\sigma, \sigma^{\prime}\right\rangle}{\Longrightarrow} S^{\prime}$ is defined in terms of the stucture of $S$. It describes an execution step of $S$ in state $\sigma$ which results in $\sigma^{\prime}$. The statement $S^{\prime}$ denotes the remaining statement to be executed. We present the following two cases (the symbol $E$ below denotes termination, the append operation is denoted by ' ', and the operation $f$ applied to a sequence gives its first element):

Output statements are described by a transition

$$
c ! ! e \stackrel{\left\langle\sigma, \sigma^{\prime}\right\rangle}{\Longrightarrow} E
$$

where $\sigma^{\prime}=\sigma\{\sigma(c ! !) \cdot \sigma(e) / c ! !\}$, i.e. the state which results from $\sigma$ by assigning to the output variable $c$ !! the sequence obtained by appending the value of $e$ (in $\sigma)$ to $\sigma(c ! !)$.

Let $\sigma(c)$ denote the suffix of $\sigma(c ! !)$ corresponding to its prefix as given by $\sigma(c ?$ ?) (so $\sigma(c)$ presents the contents of the buffer).

Input statements then are described by a transition

$$
c ? ? x \stackrel{\left\langle\sigma, \sigma^{\prime}\right\rangle}{\Longrightarrow} E
$$

provided $\sigma(c)$ is nonempty. Here $\sigma^{\prime}=\sigma\{\sigma(c ? ?) \cdot v / c ? ?, v / x\}$, where $v=$ $f(\sigma(c))$. So $\sigma^{\prime}$ results from assigning the first element to be read, i.e. $f(\sigma(c))$, to $x$ and updating the input variable $c$ ?? by appending this element to the sequence of values read sofar.

We define the semantics of a statement $S$ as the set of (finite) sequences, so-called reactive sequences, of elements of $\Sigma \times \Sigma$ generated by the above transition relation (such sequences are introduced in [4] as a general model for asynchronous communication).

Definition 4 For $w=\left\langle\sigma_{1}, \sigma_{1}^{\prime}\right\rangle \cdots\left\langle\sigma_{n}, \sigma_{n}^{\prime}\right\rangle \in(\Sigma \times \Sigma)^{*}, S \stackrel{w}{\Longrightarrow} E$ holds if there exist statements $S_{1}, \ldots, S_{n}$ such that $S_{1}=S, S_{n}=E$ and, for $1 \leq i<n$, $S_{i} \stackrel{\left\langle\sigma_{i}, \sigma_{i}^{\prime}\right\rangle}{\Longrightarrow} S_{i+1}$. For any statement $S$ with $\operatorname{Pvar}(S) \subseteq \operatorname{Pvar}_{m}$ and $\operatorname{IOvar}(S) \subseteq$ IOvar $_{m}$, the semantics $\mathcal{M}(S) \subseteq(\Sigma \times \Sigma)^{*}$ is defined by

$$
\mathcal{M}(S)=\{w \mid S \stackrel{w}{\Longrightarrow} E \text { and } w \text { is m-connected }\}
$$

where sequence $\left\langle\sigma_{1}, \sigma_{1}^{\prime}\right\rangle \cdots\left\langle\sigma_{n}, \sigma_{n}^{\prime}\right\rangle$ is $m$-connected if for all $1 \leq i<n, \sigma_{i+1}$ and $\sigma_{i}^{\prime}$ agree on all the variables in $\operatorname{Pvar}_{m}$ and $\operatorname{IOvar}_{m}$. By $\mathcal{M}(S)(\sigma)$ we denote the set of sequences $\left\langle\sigma_{1}, \sigma_{1}^{\prime}\right\rangle \cdots\left\langle\sigma_{n}, \sigma_{n}^{\prime}\right\rangle$ with $\sigma=\sigma_{1}$.

A sequence in set $\mathcal{M}(S)$ represents a computation of $S$ in some environment. The environmental steps in a sequence $\left\langle\sigma_{1}, \sigma_{1}^{\prime}\right\rangle \cdots\left\langle\sigma_{n}, \sigma_{n}^{\prime}\right\rangle$ are represented by 
the 'gaps' $\left\langle\sigma_{i}^{\prime}, \sigma_{i+1}\right\rangle$. The requirement of $m$-connectedness guarantees that the environment does not affect the (program and input/output) variables controlled by $S$.

Definition 5 For any program $P=\left[S_{1}\|\ldots\| S_{n}\right]$, the semantics $\mathcal{M}(P) \subseteq$ $\Sigma \times \Sigma$ of $P$ is defined by

$$
\mathcal{M}(P)=\alpha\left(\mathcal{M}\left(S_{1}\right)\|\ldots\| \mathcal{M}\left(S_{n}\right)\right),
$$

where the abstraction operator $\alpha$ returns the set of pairs $\left\langle\sigma, \sigma^{\prime}\right\rangle$ of initial and final states of all connected sequences, such that for all channels $c$ in the program $\sigma(c$ ?? $) \leq \sigma(c ! !)$ holds. An element $\left\langle\sigma_{1}, \sigma_{1}^{\prime}\right\rangle \cdots\left\langle\sigma_{n}, \sigma_{n}^{\prime}\right\rangle$ of $(\Sigma \times \Sigma)^{*}$ is called connected if $\sigma_{i}^{\prime}=\sigma_{i+1}$, for $1 \leq i<n$. The semantic operator $\|$ denotes the usual operation of interleaving. By $\mathcal{M}(P)(\sigma)$ we denote the set of states $\sigma^{\prime}$ such that $\left\langle\sigma, \sigma^{\prime}\right\rangle \in \mathcal{M}(P)$. (Thus, if $\sigma(c$ ??) is not a prefix of $\sigma(c ! !)$ for some channel $c$ in program $P$, then $M(P)(\sigma)=\emptyset$ holds.)

\section{PROOF SYSTEM}

In this section we present axioms and proof rules for formally reasoning about programs.

\subsection{Correctness formulas}

We assume given some logic to reason about properties of states. The vocabulary of the logic includes the set of program variables and input/output variables, and function/predicate symbols describing operations/relations on the domain $\mathrm{Val}$ of values and the domain $\mathrm{Val}^{*}$ of sequences (of values). Assertions of the underlying logic are denoted by $p, q, \ldots$ By $\sigma \vDash p$ we denote that assertion $p$ holds in $\sigma$. Validity of an assertion $p$ is denoted by $\vDash p$.

Local correctness formulas are of the form $I:\{p\} S\{q\}$, for assertions $p, q, I$ and statement $S$. Here, $I$ is an assertion which describes certain invariant properties of computations of $S$ starting in a state satisfying $p$. W.r.t. a local correctness formula $I:\{p\} S\{q\}$, it is implicitly assumed that $P \operatorname{Var}(S, p, q) \subseteq$ $\operatorname{Pvar}_{n}$ and $\operatorname{IOvar}(S, p, q) \subseteq \operatorname{IOvar}_{n}$, for some $n$. Invariant $I$ may refer to arbitrary input/output variables, but is allowed to refer only to those program variables of $S$ which occur as auxiliary variables. Informally, $I:\{p\} S\{q\}$ is valid iff

every terminating computation of $S$ in a parallel environment, which guarantees $I$ at each interleaving point, preserves $I$ and results in a state satisfying postcondition $q$, if initially $p$ holds.

Global correctness formulas are of the form $\{p\} P\{q\}$, for assertions $p, q$ and 
program $P$. The correctness formula $\{p\} P\{q\}$ holds if every terminating computation of $P$ in a state satisfying precondition $p$ results in a state satisfying postcondition $q$.

We now turn to the formal semantics of correctness formulas:

Definition 6 Let $\mathcal{M}_{\leq}(S)$ denote the prefix closure of $\mathcal{M}(S)$, i.e. it consists of $\mathcal{M}(S)$ plus all the prefixes of sequences of $\mathcal{M}(S)$. We define $\models I:\{p\} S\{q\}$ iff for every sequence $w=\left\langle\sigma_{1}, \sigma_{1}^{\prime}\right\rangle \cdots\left\langle\sigma_{n}, \sigma_{n}^{\prime}\right\rangle \in \mathcal{M}_{\leq}(S)$, such that $\sigma_{1} \vDash p$ and $\sigma_{i} \vDash I$, for $1 \leq i \leq n$, we have that $\sigma_{i}^{\prime} \vDash I$, for $1 \leq i \leq n$; and if $w \in M(S)$ then $\sigma_{n}^{\prime} \vDash q$.

Invariant $I$ in local correctness formula $I:\{p\} S\{q\}$ thus provides for a kind of rely/guarantee style specification: If the states produced by the environment, i.e. the states $\sigma_{i}, i=1, \ldots, n$, in the definition above, all satisfy the invariant $I$ then the process guarentees that $I$ is preserved by its local statechanges, i.e. $I$ holds in $\sigma_{i}^{\prime}, i=1, \ldots, n$. Note that although local correctness formulas have the same format as formulas in I-logic [20], their interpretations are different (in I-logic the invariant is used actually only for describing nonterminating behaviours, whereas in our logic the invariant is essential for compositionality).

Definition 7 For global correctness formula $\{p\} P\{q\}, \models\{p\} P\{q\}$ holds iff for all $\left\langle\sigma, \sigma^{\prime}\right\rangle \in \mathcal{M}(P)$ with $\sigma \models p$ we have that $\sigma^{\prime} \vDash q$ is satisfied.

This is the standard partial correctness interpretation of correctness formulas in Hoare logics.

\subsection{Axioms and rules}

Next we discuss the axioms and rules of the proof system. We have the following axiom for assignments:

\section{Axiom 1 (assignment) $I:\{p[e / x]\} x:=e\{q\}$}

Here $p[e / x]$ denotes the result of substituting all free occurrences of $x$ in $p$ by $e$. Since the invariant $I$ does not refer to program variables (except for the auxiliary variables, but these occur only in bracketed sections for which we have different rules), $I$ is on purely syntactic grounds preserved by the assignments.

The following axiomatization of input/output statements closely mirrors their semantics. 
Rule 1 (output) $\frac{(I \wedge p) \rightarrow(I \wedge q)[c ! ! \cdot e / c ! !]}{I:\{p\} c ! ! e\{q\}}$

The output rule thus states that the local specification $I:\{p\} c ! ! e\{q\}$ can be derived if the assertion $I \wedge p$ implies the assertion obtained from $I \wedge q$ by replacing every occurrence of the output variable $c$ !! by the sequence term $c ! ! \cdot e$. The term $c ! ! \cdot e$ records the fact that the value of $e$ has been sent.

In order to axiomatize input statements we assume in the logic a binary sequence operator '-' such that $s-s$ ' denotes the suffix of $s$ as determined by $s^{\prime}$ (in case $s^{\prime}$ is a prefix of $s$, otherwise the result is undefined) and an unary sequence operator $f$ which yields the first element of a sequence. In the rule below the term $c$ stands for $c ! !-c$ ??, i.e. it represents the contents of the buffer, the values sent but not yet read.

Rule 2 (input)

$$
\frac{(I \wedge p \wedge c \neq \epsilon) \rightarrow(I \wedge q)[f(c) / x, c ? ? \cdot f(c) / c ? ?]}{I:\{p\} c ? ? x\{q\}}
$$

The input rule thus states that the local specification $I:\{p\} c ? ? e\{q\}$ can be derived if the assertion $I \wedge p$ together with the assumption that the buffer $c$ is nonempty (formally described by $c \neq \epsilon$ ) implies the assertion obtained from $I \wedge q$ by replacing every occurrence of the variable $x$ by the term $f(c)$, which denotes by definition the first element to be read, and by replacing every occurrence of the input variable $c$ ?? by the sequence term $c ? ? \cdot f(c)$, which records that the first element of the buffer $c$ has been read.

The straightforward generalization of the above rules to bracketed sections are ommitted but can be found in the full paper.

The following rules for sequential composition, the guarded statement and the guarded iteration are the usual ones, except for the addition of invariant $I$.

Rule 3 (sequential composition) $\frac{I:\{p\} S_{1}\{r\}, I:\{r\} S_{2}\{q\}}{I:\{p\} S_{1} ; S_{2}\{q\}}$

For notational convenience we treat the case of the conditional with only guards of the form $b ; c ? ? x$.

$\begin{array}{lc}\text { Rule } 4 \text { (guarded conditional) } & \frac{I:\left\{p \wedge b_{i}\right\} c_{i} ? ? x_{i} ; S_{i}\{q\}}{I:\{p\} \rrbracket_{i}\left[b_{i} ; c_{i} ? ? x_{i} \rightarrow S_{i}\right]\{q\}} \\ \text { Rule } 5 \text { (guarded iteration) } & \frac{I:\{p\} \rrbracket_{i}\left[g_{i} \rightarrow S_{i}\right]\{p\}}{I:\{p\} \star \|_{i}\left[g_{i} \rightarrow S_{i}\right]\left\{p \wedge \wedge_{i} \neg b_{i}\right\}}\end{array}$

Moreover we have the following consequence rule. 
Rule 6 (Local consequence rule) $\frac{(I \wedge p) \rightarrow p^{\prime}, I:\left\{p^{\prime}\right\} S\left\{q^{\prime}\right\},\left(I \wedge q^{\prime}\right) \rightarrow q}{I:\{p\} S\{q\}}$

It is important to note that the rely/guarantee style of specification inherent in the interpretation of the invariant does not allow a weakening of the invariant in the above consequence rule.

We have the following simple rule for parallel composition:

Rule 7 (parallel composition) $\frac{I:\left\{p_{i}\right\} S_{i}\left\{q_{i}\right\} i=1, \ldots, n}{\left\{I \wedge \bigwedge_{i=1}^{n} p_{i}\right\}\left[S_{1}\|\ldots\| S_{n}\right]\left\{I \wedge \bigwedge_{i=1}^{n} q_{i}\right\}}$

Note that the rule does not involve any additional test on the way the local specifications of the components have been derived.

We conclude the exposition of the proof system with the global consequence rule, the auxiliary variables rule, the FIFO rule and the elimination rule.

Rule 8 (global consequence rule) $\frac{p \rightarrow p^{\prime},\left\{p^{\prime}\right\} P\left\{q^{\prime}\right\}, q^{\prime} \rightarrow q}{\{p\} P\{q\}}$

Rule 9 (auxiliary variables rule) $\frac{\{p\} P^{\prime}\{q\}}{\{p\} P\{q\}}$

where $P$ is obtained from $P^{\prime}$ by deleting all assignments to the auxiliary variables. It is assumed that the postcondition $q$ does not refer to the auxiliary variables of $P^{\prime}$.

Rule 10 (FIFO rule) $\frac{\{p \wedge c ? ? \leq c ! !\} P\{q\}}{\{p\} P\{q\}}$

where channel $c$ occurs in program $P$, and ' $\leq$ ' denotes the prefix relation on sequences.

Rule 11 (elimination rule) $\frac{\{p\} P\{q\}}{\{\exists x \cdot p\} P\{q\}}$

provided $x$ is a variable not occurring in $P$ or $q$.

The following small (toy) examples of correctness proofs illustrate some characteristic reasoning patterns of asynchronous communication. First we consider the case of a simple communication:

$$
\{c ? ?=\epsilon \wedge c ! !=\epsilon\} c ! ! e \| c ? ? x\{x=e\}
$$


This correctness formula states that after the execution of $c ! ! e \| c ? ? x$ in a state which satisfies $c ? ?=\epsilon \wedge c ! !=\epsilon$, that is, no values have been sent nor read yet, the value of $x$ equals that of $e$. We take as global invariant the assertion $c ? ? \leq c$ !!. Now, since

$$
(c ? ? \leq c ! ! \wedge c ? ?=\epsilon \wedge c \neq \epsilon) \rightarrow(c ? ? \cdot f(c) \leq c ! ! \wedge(c ? ? \cdot f(c))[1]=f(c))
$$

(given a term $t$ which denotes a sequence of values, $t[n]$, with $n$ an arithmetic expression, denotes the $n$th element of $t$ ), we have by the input rule

$$
c ? ? \leq c ! !:\{c ? ?=\epsilon\} c ? ? x\{c ? ?[1]=x\}
$$

Similarly we derive by the output rule that

$$
c ? ? \leq c ! !:\{c ! !=\epsilon\} c ! ! e\{c ! ![1]=e\}
$$

An application of the rule for parallel composition and the global consequence rule (observe the use of the global invariant $c ? ? \leq c !$ ! in the application of the consequence rule) then finishes the proof.

Next we consider the case of a computation which deadlocks:

$$
\{c=\epsilon \wedge d=\epsilon\} c ? ? x ; d ! ! e \| d ? ? y ; c ! ! e^{\prime}\{\text { false }\}
$$

We take as global invariant $I$ the precondition $c=\epsilon \wedge d=\epsilon$ : Since $(I \wedge c \neq$ $\epsilon) \rightarrow$ false (and similarly $(I \wedge d \neq \epsilon) \rightarrow$ false) we derive by the input rule

$$
I:\{\text { true }\} \text { c??x\{false }\}
$$

(and a similar correctness formula for $d ? ? y$ ). An application of the rule for sequential composition and the rule for parallel composition then finishes the proof.

The following example illustrates the use of auxiliary variables:

$$
\begin{gathered}
(\text { true } \rightarrow c ? ? x ; d ! ! 0 \square \text { true } \rightarrow d ! ! 1 ; c ? ? x) \\
\| \\
(\text { true } \rightarrow d ? ? y ; c ! ! 0 \square \text { true } \rightarrow c ! ! 1 ; d ? ? y)
\end{gathered}
$$

Assuming that all the input/output variables (c??,c!!, $d ? ?, d ! !)$ are initialized to the empty sequence, we have upon termination the following possible combinations of values of $x$ and $y$ :

$$
(x=1 \wedge y=0) \vee(x=1 \wedge y=1) \vee(x=0 \wedge y=1)
$$

That is, the combination $(x=0 \wedge y=0)$ is excluded because the two first branches of the parallel components cannot be activated during one computation. In order to prove this we need the introduction of auxiliary variables. A possible way to do so is described below:

$$
\begin{gathered}
\text { true } \rightarrow\left\langle c ? ? x ; h_{1}:=0\right\rangle ; d ! ! 0 \square \text { true } \rightarrow\left\langle d ! ! 1 ; h_{1}:=1\right\rangle ; c ? ? x \\
\text { true } \rightarrow\left\langle d ? ? y ; h_{2}:=0\right\rangle ; c ! ! 0 \square \text { true } \rightarrow\left\langle c ! ! 1 ; h_{2}:=1\right\rangle ; d ? ? y
\end{gathered}
$$


Thus the auxiliary variables $h_{1}$ and $h_{2}$ simply indicate which branches have been selected. Assuming that all the input/output variables $(c ? ?, c ! !, d ? ?, d ! !)$ are initialized to the empty sequence and that $h_{1}$ and $h_{2}$ are initialized to, say -1 , we can derive the postcondition (1), using the global invariant $I$

$$
\neg\left(h_{1}=0 \wedge h_{2}=0\right) \wedge\left(d ! !=\epsilon \rightarrow h_{2} \neq 0\right) \wedge\left(c ! !=\epsilon \rightarrow h_{1} \neq 0\right)
$$

First observe that $I$ holds initially, since $h_{1}=h_{2}=-1$ holds initially by assumption. Let us now highlight the main points in the proof of the invariance of $I$ over the branch

$$
\text { true } \rightarrow\left\langle c ? ? x ; h_{1}:=0\right\rangle ; d ! ! 0
$$

First we have to show that $I$ holds after $\left\langle c ? ? x ; h_{1}:=0\right\rangle$. Since $h_{1}$ becomes 0 we thus have to show that $h_{2}$ is different from 0 . But locally we know that $d ! !=\epsilon$, this then together with $I$ gives us that indeed $h_{2} \neq 0$. Next we have to argue that $I$ holds after $d ! ! 0$ : Just observe that $d ! ! \neq \epsilon$ holds after its execution. The proof of the invariance of $I$ over the other branches is similar. The information that $h_{1}$ and $h_{2}$ cannot be 0 at the same time now can be used to eliminate the combination $x=0 \wedge y=0$ upon termination. The involves basically the same reasoning pattern as in the above example $c ! ! e \| c ? ? x$, and is therefore omitted.

It can be shown that the last example cannot be proved correct with input/output variables only. This demonstrates that auxiliary variables (apart from the built-in input/output variables) are needed in general to reason about nondeterministic programs. In [7] however it is shown that when programs are restricted to deterministic control structures, input/output variables suffice.

\section{EXAMPLE}

We now demonstrate the applicability of our proof system by proving correct a distributed program for leader election.

Consider program $P=\left[S_{1}\|\ldots\| S_{n}\right]$ with

$$
\begin{aligned}
S_{i}=z_{i}:=0 ; y_{i}:=0 ; c_{i} ! !\left(x_{i}, 0\right) & ; \\
*\left[y_{i} \neq \infty ; c_{i-1} ? ?\left(y_{i}, h o p_{i}\right) ;\right. & {\left[x_{i}=y_{i} \rightarrow z_{i}:=1 ; y_{i}:=\infty ; c_{i} ! !(\infty, \text { hop }+1)\right.} \\
& \square \\
& x_{i}>y_{i} \rightarrow \text { skip } \\
& \square \\
& x_{i}<y_{i} \rightarrow c_{i} ! !\left(y_{i}, \text { hop }_{i}+1\right) \\
& ]
\end{aligned}
$$

Figure 1 Distributed Leader Election.

In this program, there are $n+1$ processes, $n>1$ arranged in a ring, with 
channel $c_{i}$ connecting $S_{i}$ to $S_{i+1}$. Addition and subtraction involving such indices $i$ is done modulo $n+1$. Each process $S_{i}$ has a local variable $x_{i}$ (all indices are explicit, for readability). Distinct variables $x_{i}$ and $x_{j}$ represent distinct integer values. Here ' $\infty$ ' denotes a value larger than all integers. In addition, each process has a local variable $z_{i}$ whose initial value is irrelevant. Upon termination, it is required that there exists exactly one index $i_{0}$, such that $z_{i_{0}}=1$, and $z_{j}=0$, for all $j \neq i_{0}$. The idea is to choose as the 'leader' $S_{i_{0}}$ such that $x_{i_{0}}=\max \left\{x_{k} \mid 1 \leq k \leq n\right\}$, i.e., the index of the process with the largest value of $x_{i}$.

Each process sends its value $x_{i}$ to the right, and propagates to the right any received value $x$ larger than its own $x_{i}$. The maximal value $x_{i_{0}}$ is the only one to traverse the whole ring. When it arrives back to its origin, a ' $\infty$ ' message is sent around the ring to terminate all other processes. The second component hop in message $(x, h o p)$ records the number of hops, i.e., the distance that $x$ has traversed in the ring. (This component as well as the variables $h o p_{i}, i=1, \ldots, n$, have been added only to simplify the correctness proof. Strictly speaking, they are not needed.) It is assumed that for $i=0, \cdots$, $n, x_{i}<\infty$ holds, and that $\infty$ and pairs such as $(x, h o p)$ can be encoded as natural numbers to conform to the syntax of programs.

The correctness assertion we want to establish is:

$(*) \vdash$

$$
\left\{\bigwedge_{i=0}^{n}\left(c_{i}=\epsilon \wedge x_{i}=X_{i} \neq \infty \wedge \bigwedge_{j=0}^{n}\left(j \neq i \Rightarrow X_{j} \neq X_{i}\right)\right)\right\}
$$

$$
\left\{\bigvee_{i=1}^{n}\left(z_{i}=1 \wedge \bigwedge_{j=0}^{n}\left(j \neq i \Rightarrow\left(z_{j}=0 \wedge X_{i}>X_{j}\right)\right)\right)\right\}
$$

For this example, there is no need to introduce bracketed sections.

Let $p_{i}=c_{i-1} ? ?=c_{i} ! !=\epsilon \wedge x_{i}=X_{i} \neq \infty \wedge \bigwedge_{j=0}^{n}\left(j \neq i \Rightarrow X_{j} \neq X_{i}\right)$, for $i=$ $1, \ldots, n$; and let $I=\bigwedge_{i=1}^{n} c_{i} ? ? \leq c_{i}$ !! We construct local correctness assertions for each $S_{i}$. We have:

(1)

$$
\begin{aligned}
& \left\{c_{i-1} ? ?=c_{i} ! !=\epsilon \wedge x_{i}=X_{i} \neq \infty \wedge \bigwedge_{j=0}^{n}\left(j \neq i \Rightarrow X_{j} \neq X_{i}\right)\right\} \\
& \left\{z_{i}=0 \wedge c_{i-1} ? ?=c_{i} ! !=\epsilon \wedge x_{i}=X_{i} \neq \infty \wedge \wedge_{j=0}^{n}\left(j \neq i \Rightarrow X_{j} \neq X_{i}\right)\right\}
\end{aligned}
$$

as a consequence of (assignment), and $\vdash I: y_{i}:=0$

$$
\left\{z_{i}=0 \wedge c_{i-1} ? ?=c_{i} ! !=\epsilon \wedge x_{i}=X_{i} \neq \infty \wedge \bigwedge_{j=0}^{n}\left(j \neq i \Rightarrow X_{j} \neq X_{i}\right)\right\}
$$

$$
\begin{aligned}
\left\{y_{i}=0 \wedge z_{i}=0 \wedge c_{i-1} ? ?=c_{i} ! !=\epsilon\right. & \wedge x_{i}=X_{i} \neq \infty \\
& \left.\wedge \bigwedge_{j=0}^{n}\left(j \neq i \Rightarrow X_{j} \neq X_{i}\right)\right\}
\end{aligned}
$$

as a consequence of (assignment), and

$$
\begin{aligned}
& \left\{y_{i}=0 \wedge z_{i}=0 \wedge c_{i-1} ? ?=c_{i} ! !=\epsilon \wedge x_{i}=X_{i} \neq \infty\right. \\
& c_{i} ! !\left(x_{i}, 0\right) \\
& \left.\wedge \bigwedge_{j=0}^{n}\left(j \neq i \Rightarrow X_{j} \neq X_{i}\right)\right\} \\
& \left\{y_{i}=0 \wedge z_{i}=0 \wedge c_{i-1} ? ?=\epsilon \wedge c_{i} ! !=<\left(x_{i}, 0\right)>\wedge x_{i}=X_{i} \neq \infty\right. \\
& \left.\wedge \bigwedge_{j=0}^{n}\left(j \neq i \Rightarrow X_{j} \neq X_{i}\right)\right\}
\end{aligned}
$$

as a consequence of (output). 
To proceed we, obviously, need a loop invariant $L_{i}$. It comprises a number of conjuncts formulated below. (We have named these conjuncts for convenience.) For a sequence $h$ and value $x, x \in h$ denotes that $x$ occurs in $h ; h[i]$ denotes the $i^{t h}$ element of $h$, provided that $1 \leq i \leq|h|$ holds; and last $(h)$ denotes $h[|h|]$.

Define $L_{i}$ by the conjunction of

(A) $x_{i}=X_{i} \neq \infty \wedge\left(z_{i}=0 \vee z_{i}=1\right) \wedge \bigwedge_{j=0}^{n}\left(j \neq i \Rightarrow X_{j} \neq X_{i}\right)$.

(B) $z_{i}=1 \Rightarrow y_{i}=\infty$.

(C) $\left(\exists\right.$ hop. $\left(x_{i}, h o p\right) \in c_{i-1}$ ??) $\Leftrightarrow z_{i}=1$.

(D) $\forall x$, hop. $\left((x, h o p) \in c_{i} ! ! \Rightarrow x \geq x_{i}\right)$.

(E) $\forall$ hop. $\left((\infty, h o p) \in c_{i} ! ! \Rightarrow \quad(h o p \neq 0\right.$ $\wedge\left(\quad(\infty, h o p-1) \in c_{i-1} ? ?\right.$

$\left.\left.\left.\vee\left(x_{i}, h o p-1\right) \in c_{i-1} ? ?\right)\right)\right)$.

(F) $\forall x$, hop. $\left((x, h o p) \in c_{i} ! ! \Rightarrow \quad \begin{array}{l}\left(x=x_{i} \wedge \text { hop=0 }\right) \\ \vee(x=\infty \wedge \text { hop >0 })\end{array}\right.$ $\left.\vee\left((x, h o p-1) \in c_{i-1} ? ? \wedge h o p>0\right)\right)$.

(G) $y_{i}=\infty \Rightarrow \exists h o p \geq 0$. $\left(\left(x_{i}, h o p\right) \in c_{i-1}\right.$ ?? $\vee(\infty, h o p) \in c_{i-1}$ ?? $)$.

The loop invariant is established indeed:

(4)

$$
\begin{aligned}
&\left(\begin{array}{r}
y_{i}=0 \wedge z_{i}=0 \wedge c_{i-1} ? ?=\epsilon \wedge c_{i} ! !=<\left(x_{i}, 0\right)> \\
\left.\wedge x_{i}=X_{i} \neq \infty \wedge \bigwedge_{j=0}^{n}\left(j \neq i \Rightarrow X_{i} \neq X_{j}\right)\right)
\end{array}\right. \\
& \Rightarrow L_{i} .
\end{aligned}
$$

We show in some detail that $L_{i}$ serves as an invariant for the loop in $S_{i}$. First, we introduce assertion $A L T_{i}$, holding prior to the branching points in $S_{i}$. It also comprises several numbered conjuncts. $A L T_{i}$ is the conjunction of (A), (D), and

(B') $z_{i}=0$,

(C') $\forall$ hop, $k .\left(1 \leq k<\mid c_{i-1}\right.$ ?? $\left.\mid \Rightarrow\left(x_{i}, h o p\right) \neq c_{i-1} ? ?[k]\right)$,

$\left(\mathrm{E}^{\prime}\right) \forall$ Hop. $\quad\left((\infty, h o p) \in c_{i} ! !\right.$

$$
\begin{aligned}
\Rightarrow & \quad h o p \neq 0 \\
& \wedge \exists k .\left(1 \leq k<\left|c_{i-1} ? ?\right| \wedge\left(\quad(\infty, h o p-1)=c_{i-1} ? ?[k]\right.\right.
\end{aligned}
$$$$
\left.\left.\left.\vee\left(x_{i}, h o p-1\right)=c_{i-1} ? ?[k]\right)\right)\right),
$$

$\left(\mathrm{F}^{\prime}\right) \forall x$, hop. $(x, h o p) \in c_{i} ! ! \Rightarrow \quad\left(x=x_{i} \wedge\right.$ hop=0)

$\vee(x=\infty \wedge h o p>0)$

$\vee \exists k .\left(1 \leq k<\left|c_{i-1} ? ?\right| \wedge(x, h o p-1)=c_{i-1} ? ?[k]\right.$

$$
\wedge \text { hop }>0)
$$

$\left(\mathrm{G}^{\prime}\right) y_{i}=\infty \Rightarrow \exists h o p \geq 0 . \exists k .\left(1 \leq k<\left|c_{i-1} ? ?\right|\right.$

$$
\left.\wedge\left(\left(x_{i}, h o p\right)=c_{i-1} ? ?[k] \vee(\infty, h o p)=c_{i-1} ? ?[k]\right)\right),
$$

$\left(\mathrm{H}^{\prime}\right) c_{i-1} ? ? \neq \epsilon \wedge \operatorname{last}\left(c_{i-1} ? ?\right)=\left(y_{i}, h o p_{i}\right) \wedge h o p_{i} \geq 0$. 
We note that

(5) $\vdash\left\{L_{i} \wedge y_{i} \neq \infty\right\} c_{i-1} ? ?\left(y_{i}, h o p_{i}\right)\left\{A L T_{i}\right\}$

is obtained via (input). We next consider the separate directions.

For the first direction we reason as follows:

(6) $\quad\left\{A L T_{i} \wedge x_{i}=y_{i}\right\}$

$$
\begin{aligned}
& \vdash z_{i}:=1 \\
& \quad\left\{(\mathrm{~A}) \wedge z_{i}=1 \wedge\left(C^{\prime}\right) \wedge(D) \wedge\left(E^{\prime}\right) \wedge\left(F^{\prime}\right) \wedge\left(G^{\prime}\right) \wedge\left(H^{\prime}\right) \wedge x_{i}=y_{i}\right\} .
\end{aligned}
$$

(7) $\left\{(A) \wedge z_{i}=1 \wedge\left(C^{\prime}\right) \wedge(D) \wedge\left(E^{\prime}\right) \wedge\left(F^{\prime}\right) \wedge\left(G^{\prime}\right) \wedge\left(H^{\prime}\right) \wedge x_{i}=y_{i}\right\}$

$\vdash y_{i}:=\infty$

$\left\{(A) \wedge z_{i}=1 \wedge y_{i}=\infty \wedge\left(C^{\prime}\right) \wedge(D) \wedge\left(E^{\prime}\right) \wedge\left(F^{\prime}\right) \wedge c_{i-1} ? ? \neq \epsilon\right.$

$\wedge \operatorname{last}\left(c_{i-1} ? ?\right)=\left(x_{i}, h o p_{i}\right)$

$\left.\wedge h o p_{i} \geq 0\right\}$.

(8) $\quad\left\{(A) \wedge z_{i}=1 \wedge y_{i}=\infty \wedge\left(C^{\prime}\right) \wedge(D) \wedge\left(E^{\prime}\right) \wedge\left(F^{\prime}\right) \wedge c_{i-1} ? ? \neq \epsilon\right.$

$\wedge \operatorname{last}\left(c_{i-1} ? ?\right)=\left(x_{i}, h_{o p}\right)$

$c_{i} ! !\left(\infty, h o p_{i}+1\right)$

$\left.\wedge h o p_{i} \geq 0\right\}$

$\left\{L_{i}\right\}$.

Thus, the first direction re-establishes $L_{i}$.

The second direction also re-establishes $L_{i}$, since we have

(9) $\vdash\left\{A L T_{i} \wedge x_{i}>y_{i}\right\}$ skip $\left\{L_{i}\right\}$.

Finally, we have

(10) $\left\{A L T_{i} \wedge x_{i}<y_{i}\right\} c_{i} ! !\left(y_{i}, h o p_{i}+1\right)\left\{L_{i}\right\}$.

Therefore, the third direction re-establishes $L_{i}$, too.

By combining the above local correctness assertions, we obtain that $\vdash I$ : $\left\{p_{i}\right\} S_{i}\left\{q_{i}\right\}$ holds for $q_{i}=\left(L_{i} \wedge y_{i}=\infty\right), i=1, \ldots, n$.

Application of the parallel composition rule then yields $\vdash\left\{I \wedge \wedge_{i=1}^{n} p_{i}\right\}$ $P\left\{I \wedge \bigwedge_{i=1}^{n} q_{i}\right\}$. By repeatedly applying the $F I F O$ rule, we obtain that $\vdash$ $\left\{\bigwedge_{i=1}^{n} p_{i}\right\} \stackrel{P}{P}\left\{I \wedge \bigwedge_{i=1}^{n} q_{i}\right\}$ holds.

We now derive correctness formula $\left({ }^{*}\right)$ to be established for the leader election program by application of the global consequence rule. It suffices to prove that $I \wedge \bigwedge_{i=1}^{n} q_{i}$ implies $\left\{\bigvee_{i=1}^{n}\left(z_{i}=1 \wedge \bigwedge_{j=1}^{n}\left(j \neq i \Rightarrow\left(z_{j}=0 \wedge X_{i}>X_{j}\right)\right)\right)\right\}$.

To do so, assume $I \wedge \bigwedge_{i=1}^{n} q_{i}$ holds. Since $q_{i}$ has been defined as $L_{i} \wedge y_{i}=\infty$ and $L_{i}$ has been defined as the conjunction of $(\mathrm{A}), \cdots,(\mathrm{G})$, for all $i=1, \cdots$, $n$, we get from clause $(\mathrm{G})$ that 
(i) for all $i=1, \cdots, n$, there exists some $h o p \geq 0$ with $\left(x_{i}, h o p\right) \in c_{i-1}$ ?? $\vee$ $(\infty, h o p) \in c_{i-1}$ ??.

From this we derive by reductio ad absurdum that

(ii) for some $j=1, \cdots, n$, and for some $h o p \geq 0,\left(x_{j}, h o p\right) \in c_{j-1}$ ?? holds.

Suppose that (ii) is not the case. Then, for all $j=1, \cdots, n$, and for all $h o p \geq 0,\left(x_{j}, h o p\right) \notin c_{j-1}$ ?? holds. Consider an arbitrary $k \in\{1, \cdots, n\}$. From (i) we obtain that there exists some $h o p_{0} \geq 0$, such that $\left(\infty, h o p_{0}\right) \in c_{k-1}$ ??. From $I,\left(\infty, h o p_{0}\right) \in c_{k-1}$ !! is obtained. Then (E) implies that for some hop 1 with $h o p_{0}>h o p_{1} \geq 0,\left(x_{k-1}, h o p_{1}\right) \in c_{k-2}$ ?? $\vee\left(\infty, h o p_{1}\right) \in c_{k-2}$ ?? is true. Because (ii) is assumed to be false, $\left(\infty, h o p_{1}\right) \in c_{k-2}$ ?? follows. By repeating this process ad infinitum we find an infinite decreasing sequence $h o p_{0}>h o p_{1}>\ldots$ of natural numbers, which is impossible. We conclude that (ii) is satisfied.

Now, consider an arbitrary $i_{0} \in\{1, \cdots, n\}$ such that for some hop $\geq 0,\left(x_{i_{0}}, h o p\right)$ $\in c_{i_{0}-1}$ ?? holds. From (C) we obtain that $z_{i_{0}}=1$ is true; $I$ implies that for some $h o p \geq 0,\left(x_{i_{0}}, h o p\right) \in c_{i_{0}-1}$ !! holds. Then, $x_{i_{0}} \geq x_{i_{0}-1}$ follows from (D). From (A) and $(\mathrm{F})$ we obtain that for some $h o p \geq 0,\left(x_{i_{0}}, h o p\right) \in c_{i_{0}-2}$ ?? . Continuing this way, we get $\left(x_{i_{0}}, h o p\right) \in c_{i_{0}-1} ? ? \Rightarrow \bigwedge_{1 \leq j \leq n} x_{i_{0}} \geq x_{j}$. Together with (A) this proves uniqueness of $i_{0}$; and hence the righthandside of the implication we wished to prove.

Consequently, correctness of the distributed leader election program follows from rule (Consequence). This concludes our proof, and hence this section.

\section{CONCLUSION}

We have presented a compositional Hoare logic for distributed systems in which processes conmmunicate asynchronously via (unbounded) FIFO buffers. We have applied the proof system to a distributed leader election program, in order to demonstrate the applicability of our method. It has been argued that our method is preferable over other methods presented in the literature for reasoning about asynchronously communicating systems because it allows the use of arbitrary auxiliary vriables. In the full paper the proof system is shown to be sound and (relative) complete.

We remark here that the proof system can be easily extended to reasoning about nonterminating computations by introducing global correctness formulas of the form $I:\{p\} P\{q\}$ and interpreting the invariant with respect to all the (finite prefixes of) computations including the infinite ones. Due to lack of space we refer to the full paper for the details.

In [5] it is shown that auxiliary variables are no longer needed if one restricts to parallel systems of deterministic sequential processes. Moreover, in 
[7] it shown that restricting to local nondeterminism allows for a further decomposition of the global invariant.

In the future we intend to apply our method to larger programs, using interactive proof checkers like PVS [19].

\section{ACKNOWLEDGEMENT}

We are grateful to Hagit Attiya for her help in the example proof.

\section{REFERENCES}

[1] Apt, K. R. (1981) Ten years of Hoare's logic: A survey-part I. ACM Transactions of Programming Languages and Systems, Vol. 3, No. 4., pp. 431-483.

[2] Apt, K. R. (1983) Formal justification of a proof system for communicating sequential processes. Journal of the ACM, Vol. 30, pp. 197-216.

[3] Apt, K. R., Francez, N., and de Roever, W.-P. (1980), A proof system for communicating sequential processes. ACM Transactions of Programming Languages and Systems, Vol. 2, No. 3, pp. 359-385.

[4] de Boer, F. S., Kok, J. N., Palamidessi, C., and Rutten, J. J. M. M. (1991) The failure of failures: Towards a paradigm for asynchronous communication. Proceedings of the Second International Conference on Concurrency Theory, Lecture Notes in Computer Science 527, pp. 111-126, Amsterdam, The Netherlands.

[5] de Boer, F. S. and van Hulst, M. (1994) Partial correctness of asynchronously communicating processes. Proceedings of Mathematical Foundations of Computer Science 1994.

[6] de Boer, F. S. and van Hulst, M. (1995) A compositional proof system for asynchronously communicating processes. Proceedings of Mathematics of Program Construction.

[7] de Boer, F. S. and van Hulst, M. (1996) Local nondeterminism in asynchronously communicating processes. Proceedings of Formal Methods Europe 1996.

[8] Bougé, L. (1988) On the existence of symmetric algorithms to find leaders in networks of communicating sequential processes. Acta Informatica, Vol. 25, pp. 179-201.

[9] Camp, T., Kearns, P., and Ahuja, M. (1993) Proof rules for flush channels. IEEE Transactions on Software Engineering, Vol. 19, No. 4, pp. $366-378$.

[10] Francez, N. (1992) Program verification. Addison Wesley.

[11] Francez, N. and Stomp, F. A. (1993) A proof system for asynchronously communicating processes. Technical Report 722, The Technion, Department of Computer Science. 
[12] Francez, N. and Stomp, F. A. (1994) A proof system for asynchronously communicating processes. Technical Memorandum, AT\&T Bell Laboratories, December 1994.

[13] Jones, C. B. (1981) Development methods for computer programs including a notion of interference. PhD thesis, Oxford University Computing Laboratory.

[14] Jonsson, B. (1985) A model and proof system for asynchronous networks. Proceedings of the $4^{\text {th }}$ ACM Annual Symposium on Principles of Distributed Computing, pp. 49-58.

[15] Lamport, L. (1980) The 'Hoare logic' of concurrent programs. Acta Informatica 14 (1980), pp. 21-37.

[16] Lamport, L. and Schneider, F. B. (1984) The "Hoare logic" of CSP, and all that. ACM Transactions on Programming Languages and Systems, Vol. 6, No. 2, April 1984, pp. 281-296.

[17] Levin, G. M. and Gries, D. (1981) Proof techniques for communicating sequential processes. Acta Informatica 15, pp. 281-302.

[18] Owicki, S. and Gries, D. (1976) An axiomatic proof technique for parallel programs. Acta Informatica 6, pp. 319-340.

[19] Owre, S, Rushby, J., and Shankar, N. (1992) PVS: A prototype verification system. Proceedings of the 1th Conference on Automated Deduction, Lecture Notes in Artificial Intelligence, Vol. 607, SpringerVerlag, pp. 748-752.

[20] Pandya, P. K. (1988) Compositional verification of distributed Programs. $\mathrm{PhD}$ thesis, Tata Institute of Fundamental Research, Homi Bhabha Road, Bombay 400 005, INDIA.

[21] Pandya, P. K. and Joseph, M. (1991) P-A logic - a compositional proof system for distributed programs. Distributed Computing 5, pp. 37-54.

[22] Schneider, F. B. (1982) Proof rules for message passing, logics and models of concurrent systems. NATO ASI Series, Vol. F 13, SpringerVerlag, 1985. Lecture Notes in Computer Science 190, pp. 234-254.

[23] Schlichting, R. D. and Schneider, F. B. (1984) Using message passing for distributed programming, proof rules and disciplines. ACM Transactions of Programming Languages and Systems, Vol. 6, No. 3, pp. 402-431.

[24] Soundararajan, N. (1983) Correctness proofs for CSP programs. Theoretical Computer Science, Vol. 24.

[25] Zhou, P. and Hooman, J. (1992) A proof theory for asynchronously communicating real-time systems. Proceedings of the $13^{\text {th }}$ Real-Time Systems Symposium.

[26] Zwiers, J. (1989) Compositionality, concurrency and partial correctness. Lecture Notes in Computer Science 321. 


\section{BIOGRAPHIES}

Frank de Boer: His main current research interests concern semantics and logics of parallel programming languages, real-time extensions of concurrent constraint programming, and multi-agent systems.

Nissim Frances: His research interests include formal semantics of natural language, computational linguistics, semantics of programming languages, program verification, concurrent and distributed programming, and logic programming.

Marten van Hulst: He received his $\mathrm{PhD}$ in 1995 at the University of Utrecht. In his thesis compositional verification of parallel programs Marten applied epistemic logic to the verification of CSP-like languages.

Frank Stomp: His research interests are in the areas of program verification, with an emphasis on distributed computation. He has also done work on software development. 